\title{
Progress in the treatment of acute promyelocytic leukemia: optimization and obstruction
}

\author{
Junmin Li $\cdot$ Hongming Zhu $\cdot$ Jiong Hu \\ Jianqing Mi $\cdot$ Saijuan Chen $\cdot$ Zhu Chen • \\ Zhenyi Wang
}

Received: 4 December 2013/Revised: 13 May 2014/Accepted: 20 May 2014/Published online: 18 June 2014

(c) The Japanese Society of Hematology 2014

\begin{abstract}
The past three decades have witnessed a great progress in the treatment of acute promyelocytic leukemia (APL). The current application of all-trans retinoic acid, arsenic trioxide (ATO), and anthracycline-based chemotherapies has been proved to be highly effective. Based on the risk factors of APL, optimization of the treatment emphasizes the role of ATO in induction, consolidation and maintenance therapy as a substitute to chemotherapy in low- and intermediate-risk patients, and in potential reduction of chemotherapy in high-risk group without impact on the outcome. However, early death and relapse remain obstacles to further improvement of the rates of remission and long-term survival, and the acute and chronic adverse effects of ATO should be considered for more appropriate management. Efforts should be made to more rationally obtain improved outcomes through the use of less toxic regimens.
\end{abstract}

Keywords Acute promyelocytic leukemia $\cdot$ All-trans retinoic acid · Arsenic trioxide - Optimization therapy

\section{Introduction}

Acute promyelocytic leukemia (APL) accounts for $10-15 \%$ of acute myelocytic leukemia (AML). It is characterized as an arrest of leukocyte differentiation at the promyelocyte stage, morphologically identified as M3 subtype of AML by the French-American-British (FAB)

J. Li $\cdot$ H. Zhu $\cdot$ J. Hu $\cdot$ J. Mi $\cdot$ S. Chen $\cdot$ Z. Chen

Z. Wang $(\bowtie)$

Shanghai Institute of Hematology, Rui Jin Hospital, Shanghai

Jiao Tong University School of Medicine, Shanghai, China

e-mail: 13661734438@163.com classification, and cytogenetically featured with chromosomal translocation $t(15 ; 17)(\mathrm{q} 22 ; \mathrm{q} 21)$ and the presence of PML-RAR $\alpha$ fusion gene in $98 \%$ APL patients. Moreover, a series of variant chromosomal aberrations and gene rearrangements are detected in no more than $2 \%$ of the cases [1]. APL was considered to be the most malignant form of acute leukemia for decades because of its severe bleeding tendency and high rate of early death mostly due to disseminated intravascular coagulation (DIC) or hyperfibrinolysis.

In 1973, Bernard et al. [2] reported that APL cells were particularly sensitive to daunorubicin-based chemotherapy, leading to the original front-line therapy of anthracyclines [daunorubicin(DNR), idarubicin(IDA)] plus cytarabine (Ara-C). The complete remission (CR) rate of newly diagnosed APL was improved to about $75 \%$, but the early mortality rate remained as high as $15 \%$ since the risk of coagulopathy was exacerbated during chemotherapy. Meanwhile, the median duration of remission was still relatively short with the accumulated relapse rate of $35 \%$ after 2 years of CR [3]. The late 1980s embraced a dramatic improvement in the outcome of APL treatment due to the introduction of all-trans retinoic acid (ATRA). In combination with anthracycline-based chemotherapy, ATRA has considerably improved the CR rate to $90 \%$ and overall survival (OS) to $80 \%$ with tolerable side effects. Since the 1990s, arsenic trioxide (ATO) has demonstrated a series of achievements in the clinical outcome of refractory or relapsed as well as newly diagnosed APL. Recent studies focusing on the mechanism of ATRA and ATO have suggested that they have a synergistic effect on PML$\mathrm{RAR} \alpha$ fusion protein, providing solid foundation for studies on ATRA/ATO combination therapy, which may be a promising first-line therapy in the near future [4]. In this article, we review the clinical approaches of ATRA 
and ATO in the treatment of APL, which turned APL from highly fatal to highly curable, and the optimization for the treatment of APL, as well as the hurdles that still remain controversial.

\section{Differentiation therapy of ATRA for APL}

Traditional chemotherapy has played an anti-leukemic role by inhibiting the proliferation of malignant cells or killing them with cytotoxic agents; however, the normal and functional human hematopoietic cells are also injured. As is well known that differentiation arrest is one of the main pathogenesis of malignancy, it suggested an alternative way for anti-APL treatment by inducing differentiation of promyelocytic leukemic cells.

ATRA as the single agent for APL treatment

Between 1960s and 1970s, several researchers [5, 6] had discovered the differentiation of malignant cells triggered by certain agents in and beyond the hematological field. In the early 1980s, Breitman et al. [7, 8] demonstrated that retinoic acid (RA) and 13-cis-retinoic acid (13-cis-RA) were equally effective in inducing morphological and functional maturation of HL-60 cells, a cell line derived from human acute promyelocytic leukemia. Then in vivo therapeutic trials were carried out by Flynn et al. $[9,10]$ to isolated APL patients refractory to chemotherapy, suggesting that RA and 13-cis-RA might clinically induce differentiation and maturation of APL cells as well, so as to reach CR and control coagulopathy, but the successful cases were limited.

During the early 1980s, our team from Shanghai Institute of Hematology (SIH) was also dedicating a lot of efforts to the screening of the inducers for APL differentiation. It was not until 1985, when ATRA was successfully applied to a 5-year-old APL girl who was in a critical situation after failure of chemotherapy in Shanghai Children's Hospital, that the idea of targeted therapy for APL was first introduced to the world. In 1988, Huang et al. [11] reported a remarkable outcome using ATRA as differentiation therapy in 24 APL patients (16 newly diagnosed and 8 refractory cases), of whom 23 achieved CR, and the remaining one also attained $\mathrm{CR}$ after the addition of lowdose Ara-C.

ATRA alone was efficient in lowering the rate of early death by reduction of infection due to chemotherapyinduced bone marrow suppression, DIC and/or hyperfibrinolysis. It was later confirmed by a series of hematology/ oncology centers around the world that ATRA against APL could reach a CR rate of 85-90\% [12-14]. Although it was not significantly different from chemotherapy, the long- term follow-up showed a superior clinical outcome in terms of 12-month event-free survival (EFS) by the European APL 91 Group [13] and 5-year disease-free survival (DFS) by the North American Intergroup APL Trial [14].

Subsequent studies revealed that ATRA induced terminal differentiation by specifically targeting the RAR $\alpha$ moiety of PML-RAR $\alpha$ and releasing the dominant transcription repressor, which otherwise was blocked by PML$\mathrm{RAR} \alpha$ fusion protein [15].

ATRA combined with chemotherapy as a front-line therapy for APL

In the early 1990s, an initial clinical trial in China indicated that ATRA combined with chemotherapy could yield a better outcome [16]. From then on, more randomized trials evaluated the efficacy of ATRA plus anthracycline-based chemotherapy for newly diagnosed APL (Table 1).

\section{ATRA/chemotherapy combination therapy for the induction} of $A P L$

The APL93 trial by European APL Group confirmed a better EFS and lower incidence of the differentiation syndrome $[17,18]$ with the early addition of chemotherapy to ATRA as initial therapy. The successive clinical trials, i.e., LPA96 [19], LPA 99 [20] and LPA2005 [21] conducted by the Spanish PETHEMA group, used AIDA (ATRA + IDA) regimen as induction therapy, and yielded favorable CR rates $(90,91$ and $92.5 \%$, respectively) as well as the reduction of treatment-related toxicity. The Italian GIMEMA-AIEOP [22] cooperative group also demonstrated an inspiring remission rate, especially the molecular remission (MR) rate out of a well-tolerated regimen of AIDA protocol. Based on these data, ATRA/ chemotherapy combination regimen has become the standard therapy for APL induction, with regard to a significant reduction of relapse rate compared to ATRA or chemotherapy alone, and a better control of differentiation syndrome.

\section{The role of ATRA in consolidation therapy}

Consolidation therapy is aimed to achieve MR. Though using different consolidation regimens, the clinical trials around the world all demonstrated that $2-3$ cycles of anthracycline-containing chemotherapy were appropriate.

The differentiation effect of ATRA led to a hypothesis that introducing ATRA to consolidation therapy would further reduce the relapse rate by continuous differentiation of the residual APL cells. Sanz et al. [20] concluded that for intermediate- and high-risk patients, ATRA-contained consolidation therapy (LPA99) would yield a reducing 


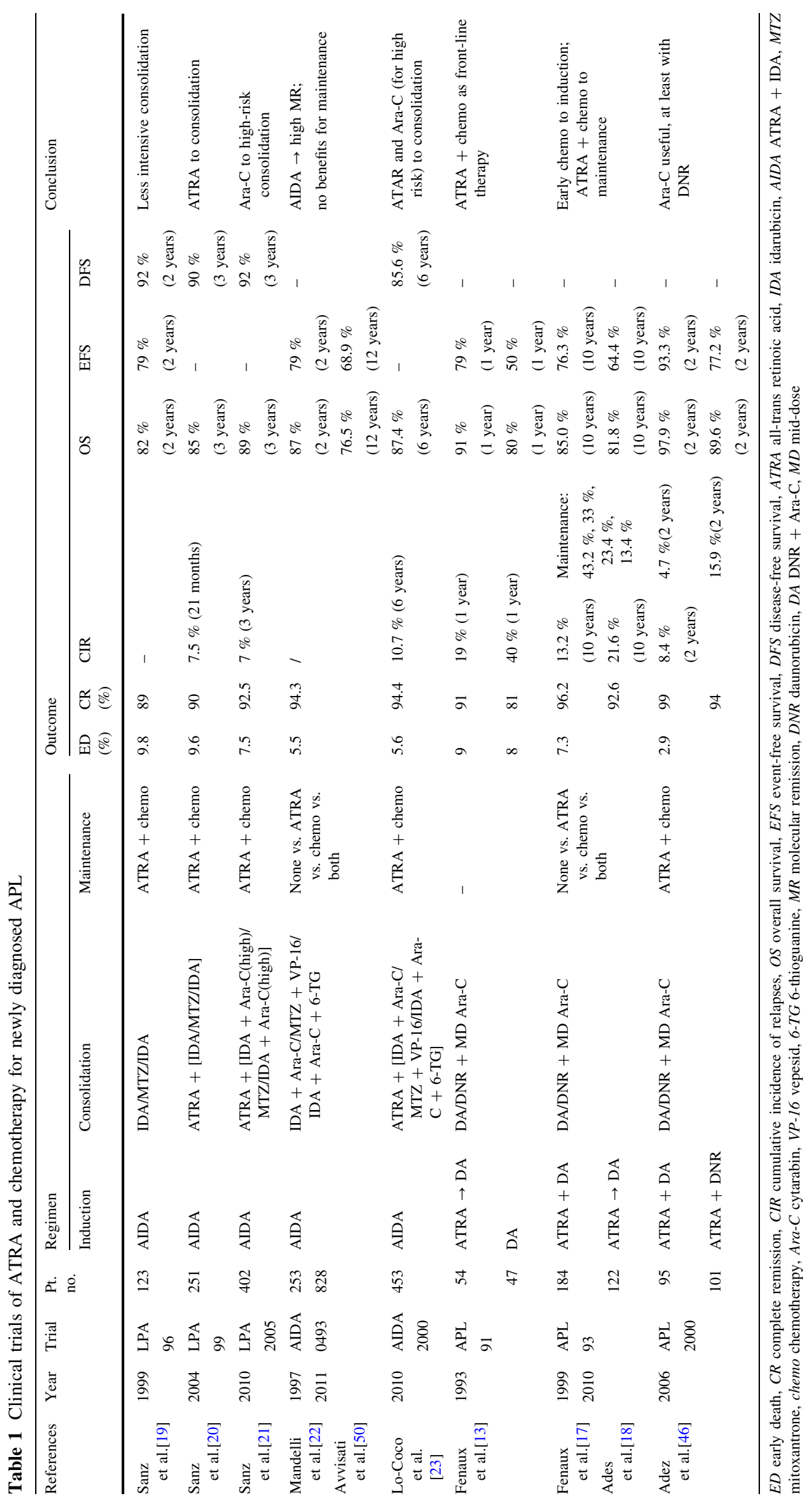


relapse rate and an improved 3-year DFS as compared to the ATRA-free LPA96 trial. Lo-Coco et al. [23] also reported a similar result when comparing ATRA-contained AIDA2000 trial with ATRA-free AIDA0493 trial. Based on these data, the National Comprehensive Cancer Network (NCCN) has recommended the addition of ATRA to consolidation therapy since 2008.

\section{Use of ATO as salvage and promising front-line therapy for APL}

The ATRA/chemotherapy front-line therapy for APL has contributed to a significant improvement in the $\mathrm{CR}$ rate and OS for APL, but relapse was still observed in 5-30 \% cases $[24,25]$. The conventional salvage therapy consisted of high-dose chemotherapy and subsequent autologous or allogeneic hematopoietic stem-cell transplantation (HSCT), which undoubtedly brought a high risk of early death and cytotoxic side effects [26]. Thus, the need for a new agent was urgent for researchers to ameliorate the prognosis and minimize the toxicity. Table 2 summarized the main trials with the application of ATO as salvage or front-line therapy for APL.

ATO as a salvage therapy for relapsed and refractory APL

Arsenic is a natural substance that has been used as medicine for over 2400 years. It was once applied to psoriasis, syphilis and chronic myelogenous leukemia as recorded by Traditional Chinese medicine (TCM) and western medicine since the eighteenth century, but suffered a precipitous decline mainly due to concerns about the toxicity and potential carcinogenicity of chronic arsenic administration in the early 1900s [27]. However, initial studies at Harbin Medical University in 1992 followed by the clinical trial at SIH in 1997 documented the remarkable remission rate achieved by ATO in patients with relapsed and newly diagnosed APL [28]. Since then, this old drug has been resuscitated by hematologists home and abroad, which perfectly demonstrated one of the principles of the ancient TCM that is "taming an evil with a toxic agent".

Following the SIH pilot trial, in 1998, Soignet et al. [29] reported a satisfactory 2nd CR (CR2) rate as well as MR rate for 12 relapsed APL patients. In the following multicenter clinical trial, they obtained a CR rate of $85 \%$ and an estimated 18-month OS and relapse-free survival (RFS) of 66 and $56 \%$, respectively [30]. According to Lengfelder's review over the 14 trials between 1997 and 2011, the single agent of ATO for relapsed APL could raise the CR2 rate up to $86 \%$ and the 2-year OS to 50-81\% [31]. Accordingly, the NCCN guideline has recommended ATO as a salvage therapy for APL patients who failed the standard therapy since 2006, and for those with little tolerance to chemotherapy since 2007 .

ATO for newly diagnosed APL, combined with ATRA as a synergistic therapy

ATO alone as induction therapy could achieve a relatively high CR rate, as demonstrated by Ghavamzadeh et al. [32, 33] and several other trials. However, ATRA was still believed to have a better control over the hemorrhagic diathesis and early death at the early stage of APL, probably due to its rapid differentiation effect. So, Shen et al. [34] at SIH proposed ATRA/ATO combination therapy (with or without chemotherapy) and observed a better CR quality compared to ATRA or ATO alone. Continued by intensive chemotherapy in consolidation and ATRA combined with ATO and low-intensity chemotherapy in maintenance, the long-term follow-up showed that the CR rate was $94.1 \%$, whereas the 5-year EFS and OS rates were 89.2 and $91.7 \%$, respectively [35]. Moreover, the use of ATRA and ATO [high-risk patients adding gemtuzumab ozogamicin (GO)] for induction and consolidation at M.D. Anderson Cancer Center also yielded pleasant results in terms of CR rate and OS [36, 37], proposing ATO as a substitute for cytotoxic agents among low-risk APL patients.

Besides the clinical analysis, the cellular and molecular mechanisms of ATO have been clarified in the subsequent studies, it exerts dose-dependent effects on APL cells, inducing the apoptosis of APL cells under high concentrations while promoting differentiation under low concentrations [38]. Both ATRA and ATO induce the degradation of PML-RAR $\alpha$ fusion protein but through distinct pathways, with ATRA targeting the RAR $\alpha$ while ATO targeting the PML moieties of the fusion protein. A synergistic effect was found in animal models without aggravating the side effects, leading to the eradication of acute promyelocytic leukemia-initiating cells (LICs) [39]. These bench-to-bedside studies well supported the clinical use of ATRA/ATO combination regimen.

In the consolidation stage, the North American Leukemia Intergroup Study C9710 [40] added two courses of ATO to the standard ATRA + DNR consolidation regimen immediately after ATRA + chemotherapy induction, displaying the superiority of ATO in consolidation with longer EFS and DFS. As for maintenance, the currently common regimen is a 1-2 year sequential administration of ATRA with or without low-intensity chemotherapy [usually 6-mercaptopurine (6-MP) and methotrexate (MTX)]. While at SIH, ATO is added to each cycle to guarantee a longer MR status, especially if chemotherapy is withdrawn in low-risk patients. Nevertheless, more randomized 


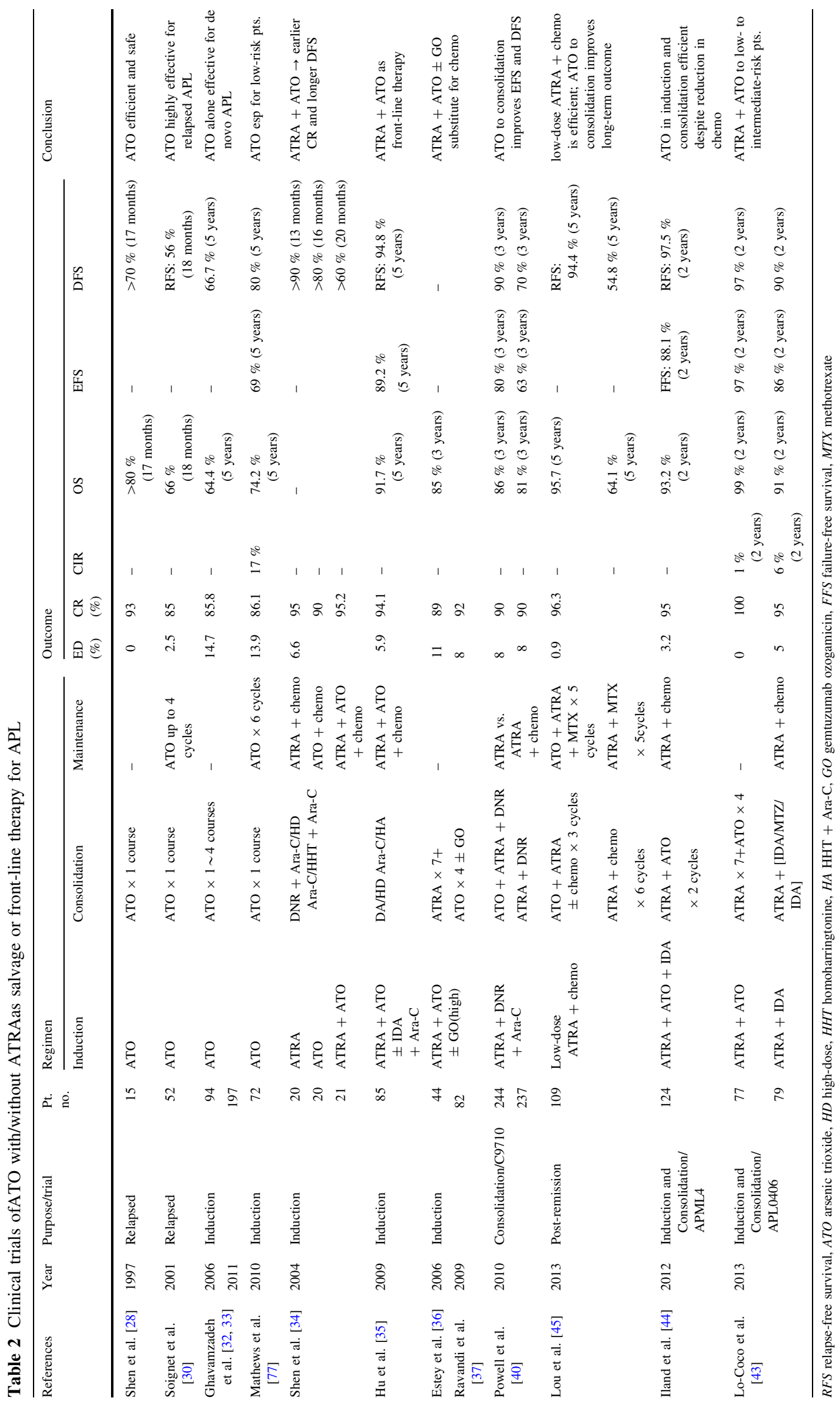


studies are expected to prove the efficacy of ATO in the maintenance therapy.

\section{Optimization in the treatment of APL}

Though scientists have achieved tremendous progress in the treatment of APL, some issues still remains controversial beyond the current standard therapy based on ATRA plus chemotherapy, especially with regards to the use of ATO and cytotoxic agents. Optimization of the treatment of APL emphasizes a personalized therapy according to the clinical characteristics of the individuals, requiring a maximal outcome not only with the most efficient and economical medications, but also with minimal adverse effects.

\section{Prognostic factors}

Prognostic factors are essential to fulfill the optimized therapy for APL. Especially for high-risk patients, they can ensure a timely intervention at the early stage, so as to reduce the occurrence of life-threatening hemorrhage and early death. The most widely accepted and practical risk factors are initial white blood cell (WBC) and platelet (PLT) counts. As reported by the Spanish PETHEMA and Italian GIMEMA cooperative groups [41], for the patients given ATRA plus IDA as induction, anthracycline-based chemotherapy as consolidation, and ATRA plus low-dose chemotherapy (6-MP and MTX) as maintenance therapy, they could be further divided into low-, intermediate-, and high-risk groups depending on the WBC and PLT counts, with $\mathrm{WBC} \leq 10 \times 10^{9} / \mathrm{L}$ and PLT $>40 \times 10^{9} / \mathrm{L}$ as lowrisk, $\quad \mathrm{WBC} \leq 10 \times 10^{9} / \mathrm{L}$ and $\mathrm{PLT} \leq 40 \times 10^{9} / \mathrm{L}$ as intermediate-risk, and $\mathrm{WBC}>10 \times 10^{9} / \mathrm{L}$ as high-risk groups, and significantly different RFS $(P<0.0001)$ was observed among the three groups.

Many other prognostic factors, such as gender, PMLRAR $\alpha$ transcript type, FLT3 mutations and CD56 [42], have also been focused on, but have not reached a consensus yet. In addition, upon ATRA/ATO combination therapy, neither the initial WBC count nor the PML-RAR $\alpha$ types or FLT3 mutations were proved to influence the prognosis of APL [35]. Furthermore, additional randomized studies should be conducted to see if the addition of ATO, especially in the maintenance phase, could ensure a better post-CR quality, so that the prognostic factors above are attenuated.

\section{Optimization of induction therapy}

The current standard induction therapy for APL is ATRA plus anthracycline-based chemotherapy. In patients who cannot tolerate chemotherapy, then ATRA/ATO induction is recommended. At SIH, however, we have been proposing the ATRA/ATO combination therapy as the front-line therapy for de novo APL for over 10 years. Although both the regimens could reach a high CR rate, ATO seems to have more advantages in the long-term follow-up.

In the APL0406 study [43], Lo-coco et al. compared the efficacy of ATRA/ATO combination with ATRA/chemotherapy combination among low- to intermediate-risk patients who were newly diagnosed with APL. The results showed that ATRA/ATO combination reached a significantly higher 2-year EFS (97.1 vs. $85.6 \%, P=0.02)$ and OS rate (98.7 vs. $91.1 \%, P=0.02)$ compared with the ATRA/chemotherapy combination, indicating that in lowto intermediate-risk APL patients, ATO may be superior to chemotherapy with less hematologic toxicities and fewer infections. This trial inspired hematologists to withdraw or at least reduce the dose of chemotherapy in low- to intermediate-risk cases.

Meanwhile, the Australian APML4 study conducted by Iland et al. [44] used the ATRA + ATO + IDA combination as induction, and 2 courses of ATRA + ATO as consolidation, also yielded higher DFS (98 vs. $86 \%$ ), freedom from relapse (FFR) and failure-free survival (FFS) rates than their former APML3 study which was ATO free. It is worth mentioning that in APML3, FLT3 mutation status was the most important predictor of OS, while in APML4, OS, FFR and FFS did not differ by FLT3 mutation status. These results were similar to our study reported by $\mathrm{Hu}$ et al. [35] in 2009.

\section{ATO in consolidation therapy}

According to the NCCN guidelines, consolidation therapy for APL generally comprises of ATRA and anthracyclinebased chemotherapy, in addition to high-dose Ara-C in high-risk patients. However, the efficacy of ATO in consolidation therapy still remains to be debated on.

Since the standard regimen may cause secondary myelodysplastic syndrome (MDS), AML or anthracyclinerelated myocardial diseases in APL patients, particularly producing unexpected events in low-risk patients who would otherwise stay in long-term $\mathrm{CR}$, more and more researchers are trying to add ATO to consolidation, in an attempt to decrease or even to suspend the use of the cytotoxic agents in the future.

The C9710 Study [40] was the first multicenter and randomized trial to emphasize the importance of ATO in consolidation therapy. Recently, Lou et al. [45] also reached the consensus that with ATO involved in post-CR period, the 6-year OS and RFS were significantly increased to 95.7 and $94.4 \%$, respectively. The APL0406 and APML4 studies are in accordance with the above 
conclusion, suggesting that repeated use of ATO might play a more important role in maintaining the relapse-free status rather than just achieving a high CR rate. The 2014 NCCN guidelines therefore took the regimen of APL0406 as category 1 recommendation for low- and intermediaterisk patients, and that of APML4 as category 2A recommendation for high-risk patients.

The success of ATO in chemotherapy-free induction and/or consolidation therapies [43, 44] provided a bright future for targeted therapy, without exposure to any conventional DNA-damaging chemotherapies in dealing with human malignancies. This will definitely inspire researchers to design more rational trials to confirm the possibility of removing chemotherapy from APL patients, or at least from low-risk group, without compromising on the clinical outcome. Further studies should pay more attention towards monitoring of MR in the era of ATRA + ATO.

Altered recognition of Ara-C in the treatment of APL

Several groups, especially the Spanish PETHEMA group, obtained high CR rates and low relapse rates in newly diagnosed APL patients treated with ATRA plus anthracyclines without Ara-C in their LPA 96 [19] and 99 [20] trials, indicating that avoiding the use of Ara-C might reduce treatment toxicity without increasing relapses.

The European APL Group then conducted a randomized trial APL2000 to evaluate the effect of Ara-C in the treatment of APL [46]. Patients $<60$ years and with standard risk (WBC count $\leq 10 \times 10^{9} / \mathrm{L}$ ) were randomized into Ara-C and no Ara-C groups in induction and consolidation therapies. The results showed that DNR alone instead of the classic DNR-Ara-C combination led to a higher risk of relapse, with the 7-year cumulative incidence of relapses (CIR) $28.6 \%$ in the non-Ara-C group, compared to $12.9 \%$ in the Ara-C group $(P=0.0065)$ [47].

The LPA99 trial, which consisted of ATRA and high cumulative dose of IDA and mitoxantrone without Ara-C in standard risk patients, obtained a lower CIR (4.2 vs. $14.3 \%, P=0.03$ ) compared to the APL2000 Ara-C group, and also less myelosuppression and therefore less mortality in CR. However, in high-risk patients, the CR rate (95.1 vs. $83.6 \%, P=0.018)$ and 3-year survival (91.5 vs. $80.8 \%, P=0.026)$ were significantly higher in the APL2000 trial, but also with an increased risk of severe infection due to myelosuppression [48].

Furthermore, the LPA2005 trial confirmed the benefits of Ara-C in reducing the relapse rate among high-risk patients, as compared to LPA99 [21]. The GEMEMA riskadapted AIDA2000 trial also involved Ara-C for consolidation therapy in high-risk patients. This suggested that anthracycline-based consolidation is at least equally effective as Ara-C-containing regimens in low-I intermediate-risk patients, and highlighted the role of Ara$\mathrm{C}$ in combination with anthracyclines and ATRA during consolidation therapy in the high-risk patients [23].

Recently, in Burnett's [49] report, the Ara-C-containing 'medical research council (MRC)' regimen did not provide any significant advantages over the ATRA/anthracycline combination (modified Spanish) regimens in terms of CR rates, 5-year OS and RFS, but with more supportive care and hospitalization. It suggested that the addition of Ara-C is not required. Although high WBC count was not a prognostic factor in this research, they did not validate the role of Ara-C in high-risk group in a prospective way.

As a matter of fact, it is generally believed that Ara-C should be avoided in low-risk patients to minimize the adverse effects of cytotoxicity. However, it should still be applied to high-risk patients to reduce the relapse rate [21].

Maintenance therapy, essential or not

It has been confirmed by several studies that maintenance therapy can further reduce the incidence of relapse in highrisk patients. On the contrary, there are still debates that early involvement of maintenance therapy may bring no benefits to low-risk patients who already attained MR [50].

The APL93 trial by the European APL Group recently updated their results, which showed that maintenance therapy using intermittent ATRA in combination with 6-MP and MTX significantly reduced 10-year CIR from $43.2 \%$ (with no maintenance) to $13.4 \%$, which is specifically effective in patients with initial WBC count higher than $5 \times 10^{9} / \mathrm{L}$ [18] . However, the Italian GIMEMA group study demonstrated recently an opposite result: there was no difference in relapses among the maintenance arms, though the randomization design was the same as in APL93 for maintenance treatment [50]. On the other hand, the Japan Adult Leukemia Study Group (JALSG) showed that there was no improvement in the DFS despite the intensified chemotherapy-based maintenance therapy [51]. In fact, the prior induction and consolidation therapies should be taken into consideration when assessing the benefits of maintenance therapy. For example, IDA involved in 2 of the above studies is probably more effective than DNR used in APL93 trial, which might cause bias when comparing their maintenance outcomes [48]. Thus, the optimization of maintenance therapy is quite variable according to different induction or consolidation treatment.

A brief summary of ATRA/ATO combination therapy at SIH and its optimization

The ATRA/ATO combination therapy for newly diagnosed APL was first conducted in 2000 at SIH. With ATRA, ATO and anthracycline-based combination for induction therapy, followed by 3 courses of chemo consolidation and 5 
cycles of sequential ATRA, ATO and low-intensity chemotherapy as maintenance, we have achieved considerably promising clinical outcomes [35].

An updated analysis with a 10-year follow-up was performed recently (the following data are unpublished). The CR rate was $91.7 \%$ (199 out of 217 patients), and the estimated 10-year OS and EFS rates were $86.3 \%$ and $78.0 \%$, respectively. While the EFS and DFS were significantly different among low-to-intermediate- and highrisk patients, the OS showed no difference, when WBC was considered as the risk factor. Given the promising results that we have attained, a randomized, open-label, multicenter trial in China is now under progress. In this study, APL patients are receiving risk-adapted optimized therapies to assess the exact role of ATO in replacing chemotherapy in low-risk patients and replacing Ara-C in highrisk patients.

\section{Thorny issues ahead of current APL treatment}

In spite of the substantial improvements achieved in the treatment of APL, there still remain several thorny challenges, mainly on the issues of early death, relapse and adverse effects.

Early death as a primary threat to APL patients

Even though timely intervention of ATRA and ATO has made enormous progress in the prognosis of APL, there are still a fair number of patients deprived of the chance to remission by early death.

Since the ATRA era, the mortality rate within induction period has been reported to be between 5 and $9 \%$ by various clinical trials [52]. As concluded by the PETHEMA LPA96 and LPA99 studies, hemorrhage accounted for $5 \%$ of early death, which was the most common cause, followed by infection $(2.3 \%)$ and APL differentiation syndrome (APLDS; $1.4 \%$ ) [53].

Severe coagulopathy usually results in an outbreak of hemorrhage in vital organs, most frequently occurring in the central nervous system (CNS), the lung and the gastrointestinal (GI) tract, and as well as the frequent incidence of thrombosis. In many instances, the death occurs within $24 \mathrm{~h}$ of life-threatening bleeding, some even before the diagnosis or enrollment of patients in the clinical trials. If cases of death before medical intervention were included, the true mortality may be higher. In Park's epidemiological study, the estimated true rate of early death was reported to be as high as $17.3 \%$ when unselected APL patients were also included, despite the wide availability of ATRA [54]. In the population-based Swedish Adult Acute Leukemia Registry trial, the rate of early death was even higher up to $29 \%$ [55]. The risk factors for the prediction of fatal hemorrhage includes high peripheral blast count $\left(>30 \times 10^{9} / \mathrm{L}\right)$, abnormal creatinine level, persistence of coagulopathy [56], initial high level of LDH, low fibrinogen level and platelet count [57]. Others reported that thrombosis may be related to high WBC count, a high level of short PML/RAR $\alpha$ isoform (bcr3), FLT-ITD, CD2 and CD15 [58]. The strategies to reduce the lethal bleeding events include early intervention of ATRA because of its effect on correction of coagulopathy by reducing the expression of tissue factor by leukemic cells, and improved supportive care consisting of the infusion of fibrinogen, platelet, and recombinant human-soluble thrombomodulin [59].

The other important early complication in the treatment of APL is the development of APLDS, which is associated with ATRA treatment but can also be observed in the early stage of ATO treatment. APLDS usually occurs within 2 weeks of induction therapy among patients with initial high WBC count or with fundamental pulmonary disorders, manifested as dyspnea with interstitial pulmonary infiltrates, unexplained fever, peripheral edema, weight gain, pleural or pericardial effusion, hypotension and acute renal failure. Once it occurs, immediate use of chemotherapy and high dose of dexamethasone can decrease the mortality rate down to $1 \%$ or lower. Prophylactic administration of corticosteroids at a dose of $10 \mathrm{mg}$ twice daily should be considered only if hyper-leukocytosis (WBC $>30 \times 10^{9} / \mathrm{L}$ ) is observed at diagnosis, or accompanied by coagulopathy [60].

Since the 1990s, the oral dose of ATRA at SIH has been modified to $25 \mathrm{mg} / \mathrm{m}^{2} /$ day to reduce the incidence of APLDS, but also to maintain the competitive edge with respect to the therapeutic efficacy as achieved by the conventional dose of $45 \mathrm{mg} / \mathrm{m}^{2} /$ day. As a consequence, the occurrence of APLDS in our patients has been very low [35], with the exception of a few patients presenting hyperleukocytosis at the diagnosis of the disease.

Relapse in APL, including CNS relapse

With the current standard treatment for APL, about 5-30\% patients demonstrated relapse [24], most of which occurred within 3 years after the termination of treatment, mainly those with high-risk factors. Prolonged time to reach the first CR, higher WBC count, previous relapses and persistent positive PML-RAR $\alpha$ after consolidation are the general risk factors for relapse [61]. Salvage treatment generally includes ATO, GO and HSCT.

The efficacy of ATO as a single agent or in combination with ATRA and chemotherapy for second remission in APL patients has been discussed above. Although yielding a high CR2 rate of approximately $86 \%$ [31] with ATO 
alone, the recurrence was not rare, as revealed by unsatisfactory DFS or RFS [30, 62]. Thus, the post-remission treatment is essential to ensure a better survival. It varies from ATO alone, ATRA + chemotherapy \pm ATO to HSCT. The European Group for Blood and Marrow Transplantation compared the leukemia-free survival between the autologous and the allogeneic HSCT groups involving 625 patients after CR1 or CR2, and found no significant differences ( 69 vs. $68 \%$ for CR1, and 51 vs. $59 \%$ for CR2). The auto-HSCT group obtained a lower transplant-related mortality but a higher incidence of relapse [63]. Most recently, a phase 2 study in Japan [64] comprised of a sequential treatment of ATO as induction and consolidation, and auto-HSCT after high-dose Ara-C chemotherapy. The findings were outstanding with a CR2 rate of $81 \%$ and 5-year EFS, and OS rates were 65 and $77 \%$, respectively. Therefore it is suggested that for relapsed APL patients, once MR is reached, the auto-HSCT should be followed, whereas allo-HSCT is strongly recommended in patients who remain molecularly positive [60].

Apart from hematological relapse, the extramedullary relapse (mainly in CNS) occurs in more than $1 \%$ of APL patients. High initial WBC count $\left(>10 \times 10^{9} / \mathrm{L}\right)$ is the most widely accepted independent risk factors for CNS relapse, with which the recurrence rate could exceed $5 \%$. Other risk factors include younger age, bcr3 PML-RAR $\alpha$ breakpoint [65] and CNS hemorrhage during induction therapy [66]. Intrathecal therapy with MTX (10-15 mg), Ara-C $(40-50 \mathrm{mg})$ and dexamethasone $(5 \mathrm{mg})$ is recommended for patients with CNS relapse. Meanwhile, ATRA and ATO combination therapy is given as systemic medication, but chemotherapy is put on hold to avoid the increasing risk of hemorrhage during lumbar puncture. For subsequent systemic chemotherapy following intrathecal intervention, high-dose Ara-C is recommended due to its high CNS penetration. Additionally, auto- or allo-HSCT and craniospinal irradiation should be considered once remission is achieved. Prophylactic intrathecal therapy, however, remains controversial in the prevention of CNS relapse. The 2012 NCCN guidelines recommended prophylactic intrathecal therapy for high-risk patients after achieving CR from induction therapy, and the guidelines in China recommend prophylactic intrathecal therapy for at least 3 times in low- and intermediate-risk patients, and 6 times in high-risk patients.

Novel agents in the treatment of APL as possible options

Novel treatment options include monoclonal antibodies and other forms or administration of arsenicals. As an antiCD33 monoclonal antibody that could specifically target the CD33 antigen expressed on the surface of APL cells, GO was approved by the US FDA in the treatment of elderly patients with relapsed APL. Lo-Coco et al. [67] treated 16 patients relapsed at the molecular level with GO as a single agent. MR was attained in 14 patients, and 7 remained in sustained MR for a median of 15 months, indicating that GO was highly effective in patients with molecular relapse, as well as in those with very advanced disease. A pilot study conducted by Ravandi et al. [37] introduced GO to de novo APL patients, proving ATRA + ATO + GO to be effective and safe, and suggesting GO as a substitute for chemotherapy-containing regimens. In conclusion, GO can be used as a single agent for patients with relapse, ATO resistance, and/or intolerance to chemotherapy due to very advanced diseases [68, 69].

Realgar-Indigo naturalis formula (RIF), also known as Compound Huangdai Tablet (CHDT), is a mixed compound of 4 agents in traditional Chinese medicine. Though the mechanism is yet to be clarified, tetra-arsenic tetrasulfide $\left(\mathrm{As}_{4} \mathrm{~S}_{4}\right)$ in realgar is considered to be the main effective agent in RIF. The oral administration of RIF saves the inconvenience of hospitalization compared with intravenous ATO. Several clinical trials conducted in China have reported the efficacy of RIF in induction, consolidation or maintenance therapy [70-72]. In a randomly assigned trial with ATRA plus RIF or ATRA plus MTX and 6-MP as maintenance therapy for APL patients in molecular remission, the 5-year RFS was significantly improved in the CHDT group (84.4 vs. $63.2 \%, P<0.05$ ) [72]. A multicenter randomized controlled trial was conducted in China to test the efficacy and safety of oral RIF compared with intravenous ATO as both induction and maintenance therapies for newly diagnosed APL. The results showed that oral RIF plus ATRA is not inferior to intravenous ATO plus ATRA as first-line treatment for APL, since there were no significant differences in the CR rate, 2-year DFS, 3-year OS or adverse effects. RIF may be considered as a routine treatment option for appropriate patients [73].

Another feasible modification is the oral formulation of ATO instead of intravenous administration. A clinical trial in Hong Kong yielded a favorable outcome when practicing oral ATO-based maintenance therapy, with the 3-year leukemia-free survival, EFS and OS at 87.7, 83.7, and $90.6 \%$, respectively [74]. An Australian single center study also demonstrated that bioavailability of oral ATO was comparable with that of intravenous administration, and similar outcomes were achieved [75]. Despite the advantages of equal efficacy, improved tolerance and compliance, the acute and chronic adverse effects of oral ATO, especially GI toxicity, must be considered and evaluated appropriately. 
FLT-3 inhibitors are also a future strategy for APL, which may be an option for post-transplant maintenance, or even obviate the need for autologous transplantation [24].

\section{Acute and chronic adverse effects of ATO}

The common acute adverse effects of ATO include APLDS (7-35 \%), leukocytosis (32-73\%), abnormal ECG changes and hepatic toxicity. Other side effects such as mild peripheral neuropathy, GI disorders, hypokalemia, hyperglycemia, neutropenia, thrombopenia, fever, headache and skin rashes [31] are also observed. APLDS, which has been discussed above, is considered to be the most severe side effect of ATO. However, the combination use of ATRA and ATO does not increase the incidence of APLDS [34]. Leukocytosis is more likely to occur in patients with initial high WBC count [76]. Therefore in recent clinical trials, hydroxyurea or anthracycline-based chemotherapy is strongly recommended in patients associated with ATOinduced leukocytosis [77].

Abnormalities in ECG may be presented as prolonged Q-Tc intervals [30, 37], T wave changes, paroxysmal supraventricular tachycardia [77], and fatal ventricular tachycardia of the torsade de pointes type [30]. Hypokalemia and hypomagnesemia may be the risk factors for cardiac events [78]. Therefore, regular monitoring of ECG and maintaining the electrolyte balance are required during the treatment.

Hepatotoxicity is widely observed in most clinical trials with the incidence rate of $33-75 \%$ [35, 77]. In the APL0406 trial, the incidence of grade 3-4 hepatotoxicity was up to $57 \%$ in the ATRA + ATO group, which was significantly higher compared with the ATRA + chemotherapy group [43]. In comparison, in our report, increased liver enzymes are quite common $(75 \%)$ but mostly mild and reversible [35]. Interestingly, we also noticed that in majority of the patients with hepatotoxicity due to first time exposure to ATO, the liver function was almost normal since the second administration of ATO.

The assessments for chronic adverse effects of intravenous ATO in APL patients are limited because of a relatively short follow-up and a small number of patients. However, as has long been acknowledged in chemistry and toxicology, chronic arsenic intoxication might induce lesions to multiple organs and systems, involving the liver, skin, cardiovascular, neurologic and GI systems [79, 80], and the most frightful adverse effect is carcinogenesis occurring specifically on the skin, lungs and bladder [80]. Smith et al. [81] estimated that the lifetime risk of dying from cancer generated by daily ingestion of 11 of water containing $50 \mu \mathrm{g} / \mathrm{l}$ arsenic could be as high as 13 per 1000 people exposed. We have long been dedicated to the evaluation of chronic arsenic-related toxicities in APL patients treated at our center, not only for the functions of organs, but also for the retention of ATO in our patients. The 5-year follow-up indicated no carcinoma, skin lesions, organ disorders or high retention of ATO after the termination of treatment [35] . However, in the most lately 10-year follow-up, we have revealed significantly higher incidence of hepatic steatosis $(42.9 \%)$ as well as chronic hepatic dysfunction $(15.2 \%)$ among these patients, although the arsenic concentration in their plasma, urine, hair and nails was not higher compared to the healthy controls (data unpublished). As a consequence, the acute and chronic hepatic toxicities due to ATO and its mechanisms as well as solutions have become the key points in our recent research. Yet, we still believe that ATO is of general long-time safety by low-dose intravenous administration for intermittent $2-5$ cycles according to the NCCN guideline.

\section{Conclusion}

In summary, APL has turned from highly fatal to highly curable subtype of AML due to the application of ATRA and ATO. Current front-line treatment consists of ATRA and anthracycline-based chemotherapy as induction and consolidation therapy, and ATRA combined with low-dose chemotherapy as maintenance therapy. ATO is recommended as the best option for relapsed APL followed by HSCT. In some of the clinical trials, ATO has been suggested as one of the first-line agents. In order to achieve a more favorable outcome with minimized toxicity, riskadapted optimization for APL is underway, especially in terms of adding ATO and/or omitting Ara-C. Apart from the improved remission rate and long-term survival, management for early death, relapse and adverse effects remain as important tasks in the future.

\section{Reference}

1. Wang ZY, Chen Z. Acute promyelocytic leukemia: from highly fatal to highly curable. Blood. 2008;111:2505-15.

2. Bernard J, Weil M, Boiron M, et al. Acute promyelocytic leukemia: results of treatment by daunorubicin. Blood. 1973;41:489-96.

3. Degos L. The history of acute promyelocytic leukaemia. Br J Haematol. 2003;122:539-53.

4. Zhou GB, Zhang J, Wang ZY, et al. Treatment of acute promyelocytic leukaemia with all-trans retinoic acid and arsenic trioxide: a paradigm of synergistic molecular targeting therapy. Philos Trans R Soc Lond B Biol Sci. 2007;362:959-71.

5. Schubert D, Humphreys S, Jacob F, et al. Induced differentiation of a neuroblastoma. Dev Biol. 1971;25:514-46.

6. Sachs L. Control of normal cell differentiation and the phenotypic reversion of malignancy in myeloid leukaemia. Nature. 1978;274:535-9.

7. Breitman TR, Selonick SE, Collins SJ. Induction of differentiation of the human promyelocytic leukemia cell line (HL-60) by retinoic acid. Proc Natl Acad Sci USA. 1980;77:2936-40. 
8. Breitman TR, Collins SJ, Keene BR. Terminal differentiation of human promyelocytic leukemic cells in primary culture in response to retinoic acid. Blood. 1981;57:1000-4.

9. Flynn PJ, Miller WJ, Weisdorf DJ, et al. Retinoic acid treatment of acute promyelocytic leukemia: in vitro and in vivo observations. Blood. 1983;62:1211-7.

10. Daenen S, Vellenga E, van Dobbenburgh OA, et al. Retinoic acid as antileukemic therapy in a patient with acute promyelocytic leukemia and Aspergillus pneumonia. Blood. 1986;67:559-61.

11. Huang ME, Ye YC, Chen SR, et al. Use of all-trans retinoic acid in the treatment of acute promyelocytic leukemia. Blood. 1988;72:567-72.

12. Wang ZY, Sun GL, Lu JX, et al. Treatment of acute promyelocytic leukemia with all-trans retinoic acid in China. Nouv Rev Fr Hematol. 1990;32:34-6.

13. Fenaux P, Le Deley MC, Castaigne S, et al. Effect of all transretinoic acid in newly diagnosed acute promyelocytic leukemia. Results of a multicenter randomized trial. European APL 91 Group. Blood. 1993;82:3241-9.

14. Tallman MS, Andersen JW, Schiffer CA, et al. All-trans retinoic acid in acute promyelocytic leukemia: long-term outcome and prognostic factor analysis from the North American Intergroup protocol. Blood. 2002;100:4298-302.

15. Mi J. Current treatment strategy of acute promyelocytic leukemia. Front Med. 2011;5:341-7.

16. Sun GL, Huang YG, Chang XF, et al. Clinical study of the treatment with all-trans retinoic acid in 544 APL patients. Chin J Hematol. 1992;13:135-7.

17. Fenaux P, Chastang C, Chevret $S$, et al. A randomized comparison of all transretinoic acid (ATRA) followed by chemotherapy and ATRA plus chemotherapy and the role of maintenance therapy in newly diagnosed acute promyelocytic leukemia. The European APL Group. Blood. 1999;94:1192-200.

18. Ades L, Guerci A, Raffoux E, et al. Very long-term outcome of acute promyelocytic leukemia after treatment with all-trans retinoic acid and chemotherapy: the European APL Group experience. Blood. 2010;115:1690-6.

19. Sanz MA, Martin G, Rayon C, et al. A modified AIDA protocol with anthracycline-based consolidation results in high antileukemic efficacy and reduced toxicity in newly diagnosed PML/ RARalpha-positive acute promyelocytic leukemia. PETHEMA group. Blood. 1999;94:3015-21.

20. Sanz MA, Martin G, Gonzalez M, et al. Risk-adapted treatment of acute promyelocytic leukemia with all-trans-retinoic acid and anthracycline monochemotherapy: a multicenter study by the PETHEMA group. Blood. 2004;103:1237-43.

21. Sanz MA, Montesinos P, Rayon C, et al. Risk-adapted treatment of acute promyelocytic leukemia based on all-trans retinoic acid and anthracycline with addition of cytarabine in consolidation therapy for high-risk patients: further improvements in treatment outcome. Blood. 2010;115:5137-46.

22. Mandelli F, Diverio D, Avvisati G, et al. Molecular remission in PML/RAR alpha-positive acute promyelocytic leukemia by combined all-trans retinoic acid and idarubicin (AIDA) therapy. Gruppo Italiano-Malattie Ematologiche Maligne dell'Adulto and Associazione Italiana di Ematologia ed Oncologia Pediatrica Cooperative Groups. Blood. 1997;90:1014-21.

23. Lo-Coco F, Avvisati G, Vignetti M, et al. Front-line treatment of acute promyelocytic leukemia with AIDA induction followed by risk-adapted consolidation for adults younger than 61 years: results of the AIDA-2000 trial of the GIMEMA Group. Blood. 2010;116:3171-9.

24. Tallman MS. Treatment of relapsed or refractory acute promyelocytic leukemia. Best Pract Res Clin Haematol. 2007;20:57-65.

25. Warrell RP Jr, de The H, Wang ZY, et al. Acute promyelocytic leukemia. N Engl J Med. 1993;329:177-89.
26. Thomas X, Dombret H, Cordonnier C, et al. Treatment of relapsing acute promyelocytic leukemia by all-trans retinoic acid therapy followed by timed sequential chemotherapy and stem cell transplantation. APL Study Group. Acute promyelocytic leukemia. Leukemia. 2000;14:1006-13.

27. Waxman S, Anderson KC. History of the development of arsenic derivatives in cancer therapy. Oncologist. 2001;6(Suppl 2):3-10.

28. Shen ZX, Chen GQ, Ni JH, et al. Use of arsenic trioxide (As2O3) in the treatment of acute promyelocytic leukemia (APL): II. Clinical efficacy and pharmacokinetics in relapsed patients. Blood. 1997;89:3354-60.

29. Soignet SL, Maslak P, Wang ZG, et al. Complete remission after treatment of acute promyelocytic leukemia with arsenic trioxide. N Engl J Med. 1998;339:1341-8.

30. Soignet SL, Frankel SR, Douer D, et al. United States multicenter study of arsenic trioxide in relapsed acute promyelocytic leukemia. J Clin Oncol. 2001;19:3852-60.

31. Lengfelder E, Hofmann WK, Nowak D. Impact of arsenic trioxide in the treatment of acute promyelocytic leukemia. Leukemia. 2012;26:433-42.

32. Ghavamzadeh A, Alimoghaddam K, Ghaffari SH, et al. Treatment of acute promyelocytic leukemia with arsenic trioxide without ATRA and/or chemotherapy. Ann Oncol. 2006;17:131-4.

33. Ghavamzadeh A, Alimoghaddam K, Rostami S, et al. Phase II study of single-agent arsenic trioxide for the front-line therapy of acute promyelocytic leukemia. J Clin Oncol. 2011;29:2753-7.

34. Shen ZX, Shi ZZ, Fang J, et al. All-trans retinoic acid/As2O3 combination yields a high quality remission and survival in newly diagnosed acute promyelocytic leukemia. Proc Natl Acad Sci USA. 2004;101:5328-35.

35. Hu J, Liu YF, Wu CF, et al. Long-term efficacy and safety of alltrans retinoic acid/arsenic trioxide-based therapy in newly diagnosed acute promyelocytic leukemia. Proc Natl Acad Sci USA. 2009;106:3342-7.

36. Estey E, Garcia-Manero G, Ferrajoli A, et al. Use of all-trans retinoic acid plus arsenic trioxide as an alternative to chemotherapy in untreated acute promyelocytic leukemia. Blood. 2006;107:3469-73.

37. Ravandi F, Estey E, Jones D, et al. Effective treatment of acute promyelocytic leukemia with all-trans-retinoic acid, arsenic trioxide, and gemtuzumab ozogamicin. J Clin Oncol. 2009;27:504-10

38. Chen GQ, Shi XG, Tang W, et al. Use of arsenic trioxide (As2O3) in the treatment of acute promyelocytic leukemia (APL): I. As2O3 exerts dose-dependent dual effects on APL cells. Blood. 1997;89:3345-53.

39. Nasr R, Guillemin MC, Ferhi O, et al. Eradication of acute promyelocytic leukemia-initiating cells through PML-RARA degradation. Nat Med. 2008;14:1333-42.

40. Powell BL, Moser B, Stock W, et al. Arsenic trioxide improves event-free and overall survival for adults with acute promyelocytic leukemia: North American Leukemia Intergroup Study C9710. Blood. 2010;116:3751-7.

41. Sanz MA, Lo Coco F, Martin G, et al. Definition of relapse risk and role of nonanthracycline drugs for consolidation in patients with acute promyelocytic leukemia: a joint study of the PETHEMA and GIMEMA cooperative groups. Blood. 2000;96:1247-53.

42. Ito S, Ishida Y, Oyake T, et al. Clinical and biological significance of CD56 antigen expression in acute promyelocytic leukemia. Leuk Lymphoma. 2004;45:1783-9.

43. Lo-Coco F, Avvisati G, Vignetti M, et al. Retinoic acid and arsenic trioxide for acute promyelocytic leukemia. N Engl J Med. 2013;369:111-21. 
44. Iland HJ, Bradstock K, Supple SG, et al. All-trans-retinoic acid, idarubicin, and IV arsenic trioxide as initial therapy in acute promyelocytic leukemia (APML4). Blood. 2012;120:1570-80 quiz 1752.

45. Lou YJ, Jin J. Long-term efficacy of low-dose all-trans retinoic acid plus individually adapted chemotherapy induction followed by arsenic trioxide based post-remission therapy in adults with newly diagnosed acute promyelocytic leukemia. Blood (ASH Annual Meeting Abstracts). 2012;120:1480.

46. Ades L, Chevret S, Raffoux E, et al. Is cytarabine useful in the treatment of acute promyelocytic leukemia? Results of a randomized trial from the European Acute Promyelocytic Leukemia Group. J Clin Oncol. 2006;24:5703-10.

47. Ades L, Chevret S, Raffoux E, et al. Long-term follow-up of European APL 2000 trial, evaluating the role of cytarabine combined with ATRA and Daunorubicin in the treatment of nonelderly APL patients. Am J Hematol. 2013;88:556-9.

48. Ades L, Sanz MA, Chevret S, et al. Treatment of newly diagnosed acute promyelocytic leukemia (APL): a comparison of French-Belgian-Swiss and PETHEMA results. Blood. 2008;111:1078-84.

49. Burnett AK, Hills RK, Grimwade D, et al. Inclusion of chemotherapy in addition to anthracycline in the treatment of acute promyelocytic leukaemia does not improve outcomes: results of the MRC AML15 trial. Leukemia. 2013;27:843-51.

50. Avvisati G, Lo-Coco F, Paoloni FP, et al. AIDA 0493 protocol for newly diagnosed acute promyelocytic leukemia: very longterm results and role of maintenance. Blood. 2011;117:4716-25.

51. Asou N, Kishimoto Y, Kiyoi H, et al. A randomized study with or without intensified maintenance chemotherapy in patients with acute promyelocytic leukemia who have become negative for PML-RARalpha transcript after consolidation therapy: the Japan Adult Leukemia Study Group (JALSG) APL97 study. Blood. 2007;110:59-66.

52. Tallman MS, Altman JK. How I treat acute promyelocytic leukemia. Blood. 2009;114:5126-35.

53. de la Serna J, Montesinos P, Vellenga E, et al. Causes and prognostic factors of remission induction failure in patients with acute promyelocytic leukemia treated with all-trans retinoic acid and idarubicin. Blood. 2008;111:3395-402.

54. Park JH, Qiao B, Panageas KS, et al. Early death rate in acute promyelocytic leukemia remains high despite all-trans retinoic acid. Blood. 2011;118:1248-54.

55. Lehmann S, Ravn A, Carlsson L, et al. Continuing high early death rate in acute promyelocytic leukemia: a population-based report from the Swedish Adult Acute Leukemia Registry. Leukemia. 2011;25:1128-34.

56. Choudhry A, DeLoughery TG. Bleeding and thrombosis in acute promyelocytic leukemia. Am J Hematol. 2012;87:596-603.

57. Kim DY, Lee JH, Lee JH, et al. Significance of fibrinogen, D-dimer, and LDH levels in predicting the risk of bleeding in patients with acute promyelocytic leukemia. Leuk Res. 2011;35:152-8.

58. Breccia M, Avvisati G, Latagliata R, et al. Occurrence of thrombotic events in acute promyelocytic leukemia correlates with consistent immunophenotypic and molecular features. Leukemia. 2007;21:79-83.

59. Matsushita T, Watanabe J, Honda G, et al. Thrombomodulin alfa treatment in patients with acute promyelocytic leukemia and disseminated intravascular coagulation: a retrospective analysis of an open-label, multicenter, post-marketing surveillance study cohort. Thromb Res. 2014;133:772-81.

60. Mi JQ, Li JM, Shen ZX, et al. How to manage acute promyelocytic leukemia. Leukemia. 2012;26:1743-51.

61. Estey EH. Treatment options for relapsed acute promyelocytic leukaemia. Best Pract Res Clin Haematol. 2003;16:521-34.
62. Alimoghaddam K, Ghavamzadeh A, Jahani M, et al. Treatment of relapsed acute promyelocytic leukemia by arsenic trioxide in Iran. Arch Iran Med. 2011;14:167-9.

63. Sanz MA, Labopin M, Gorin NC, et al. Hematopoietic stem cell transplantation for adults with acute promyelocytic leukemia in the ATRA era: a survey of the European Cooperative Group for Blood and Marrow Transplantation. Bone Marrow Transplant. 2007;39:461-9.

64. Yanada M, Tsuzuki M, Fujita H, et al. Phase 2 study of arsenic trioxide followed by autologous hematopoietic cell transplantation for relapsed acute promyelocytic leukemia. Blood. 2013;121:3095-102.

65. de Botton S, Sanz MA, Chevret S, et al. Extramedullary relapse in acute promyelocytic leukemia treated with all-trans retinoic acid and chemotherapy. Leukemia. 2006;20:35-41.

66. Montesinos P, Diaz-Mediavilla J, Deben G, et al. Central nervous system involvement at first relapse in patients with acute promyelocytic leukemia treated with all-trans retinoic acid and anthracycline monochemotherapy without intrathecal prophylaxis. Haematologica. 2009;94:1242-9.

67. Lo-Coco F, Cimino G, Breccia M, et al. Gemtuzumab ozogamicin (Mylotarg) as a single agent for molecularly relapsed acute promyelocytic leukemia. Blood. 2004;104:1995-9.

68. Breccia M, Cimino G, Diverio D, et al. Sustained molecular remission after low dose gemtuzumab-ozogamicin in elderly patients with advanced acute promyelocytic leukemia. Haematologica. 2007;92:1273-4.

69. Yago K, Aono M, Shimada H. Molecular remission induced by gemtuzumab ozogamicin in an elderly patient with relapsed acute promyelocytic leukemia. Rinsho Ketsueki. 2010;51:286-90.

70. Xiang Y, Wang XB, Sun SJ, et al. Compound huangdai tablet as induction therapy for 193 patients with acute promyelocytic leukemia. Zhonghua Xue Ye Xue Za Zhi. 2009;30:440-2.

71. Xiang Y, Chang XH, Cheng YB. Effect of post-remission therapy mainly with compound huangdai tablet on long-term survival of patients with acute promyelocytic leukemia. Zhongguo Zhong Xi Yi Jie He Za Zhi. 2010;30:1253-6.

72. Gong JX, Meng JB, Ma Y. Effect of post-remission therapy mainly with compound huangdai tablet on long-term survival of patients with acute promyelocytic leukemia. Zhongguo Zhong Xi Yi Jie He Za Zhi. 2012;32:1473-6.

73. Zhu HH, Wu DP, Jin J, et al. Oral tetra-arsenic tetra-sulfide formula versus intravenous arsenic trioxide as first-line treatment of acute promyelocytic leukemia: a multicenter randomized controlled trial. J Clin Oncol. 2013;31:4215-21.

74. Au WY, Kumana CR, Lee HK, et al. Oral arsenic trioxide-based maintenance regimens for first complete remission of acute promyelocytic leukemia: a 10-year follow-up study. Blood. 2011;118:6535-43.

75. Firkin F. Oral administration of arsenic trioxide in the treatment of acute promyelocytic leukaemia and accelerated phase chronic myeloid leukaemia: an Australian single-centre study. Intern Med J. 2012;42:948-52.

76. Camacho LH, Soignet SL, Chanel S, et al. Leukocytosis and the retinoic acid syndrome in patients with acute promyelocytic leukemia treated with arsenic trioxide. J Clin Oncol. 2000;18:2620-5.

77. Mathews V, George B, Chendamarai E, et al. Single-agent arsenic trioxide in the treatment of newly diagnosed acute promyelocytic leukemia: long-term follow-up data. J Clin Oncol. 2010;28:3866-71.

78. Raghu KG, Yadav GK, Singh R, et al. Evaluation of adverse cardiac effects induced by arsenic trioxide, a potent anti-APL drug. J Environ Pathol Toxicol Oncol. 2009;28:241-52.

79. Mumford JL, Wu K, Xia Y, et al. Chronic arsenic exposure and cardiac repolarization abnormalities with QT interval 
prolongation in a population-based study. Environ Health Perspect. 2007;115:690-4.

80. Jomova K, Jenisova Z, Feszterova M, et al. Arsenic: toxicity, oxidative stress and human disease. J Appl Toxicol. 2011;31:95-107.
81. Smith AH, Hopenhayn-Rich C, Bates MN, et al. Cancer risks from arsenic in drinking water. Environ Health Perspect. 1992;97:259-67. 